\title{
Possible enhancement of superconductivity in ladder-type cuprates by longitudinal compression
}

\author{
Hikaru Sakamoto and Kazuhiko Kuroki \\ Department of Physics, Osaka University, 1-1 Machikaneyama-cho, Toyonaka, Osaka 560-0043, Japan
}

(Received 5 December 2019; revised manuscript received 18 May 2020; accepted 22 May 2020; published 9 June 2020)

\begin{abstract}
We study theoretically the effect of uniaxial deformation of ladder-type cuprate superconductors. Model construction based on first principles calculation shows that the rung-to-leg ratio of the nearest-neighbor hoppings counterintuitively increases when the lattice is compressed in the longitudinal (leg) direction. This leads to an enhancement of the superconducting transition temperature, which intuitively is expected when compressed in the rung direction. Such a trend is traced back to the on-site hybridization between $\mathrm{Cu} 4 s$ and $\mathrm{Cu} d_{x^{2}-y^{2}}$ orbitals, which varies and changes sign upon lattice deformation.
\end{abstract}

DOI: 10.1103/PhysRevResearch.2.022055

Superconductivity in ladder-type cuprates has been studied extensively both theoretically and experimentally following the seminal proposal by Dagotto and Rice [1-3]. In fact, the $(\mathrm{Sr}, \mathrm{Ca}){ }_{14} \mathrm{Cu}_{24} \mathrm{O}_{41}$ compound [4], which consists of two-leg ladders and chains, were found to be superconducting with a transition temperature $T_{c}$ above $10 \mathrm{~K}$ under high pressure [5]. Theoretically, it was suggested that a stronger spin-spin coupling in the rung direction enhances superconductivity [1,6-10].

Nowadays, there is a renewed interest in the problem of ladder-type materials since a two-leg ladder lattice can be viewed as a two-band system where wide and narrow bands coexist [11-16]. In such a system, when the Fermi level is placed in the vicinity of the narrow-band edge, strong enhancement of superconductivity is expected. A band positioned in the vicinity of the Fermi level is often referred to as an "incipient band," which has attracted increased interest recently in the context of the iron-based superconductors [17-26] and also flat-band superconductivity [27-30]. In fact, as explained in more detail later, when electrons are doped in the two-leg ladder system, larger electron hopping in the rung direction pushes down the lower band so as to make this band closer to the incipient band situation, thereby enhancing superconductivity $[15,16]$.

Hence, in the two-leg ladder system, both from realspace and momentum-space viewpoints, increasing the electron hopping in the rung direction is expected to enhance superconductivity. We note that larger rung-direction hopping enhancing superconductivity is a tendency not expected in the layered cuprates, where the $\mathrm{Cu}$ atoms form a square lattice within the $\mathrm{CuO}_{2}$ layers. This is because, in real space, $x$ and $y$ directions are equivalent for the square lattice, so that the $d_{x^{2}-y^{2}}$-wave pairs are formed with equal amplitudes in the

Published by the American Physical Society under the terms of the Creative Commons Attribution 4.0 International license. Further distribution of this work must maintain attribution to the author(s) and the published article's title, journal citation, and DOI. $x$ and $y$ directions. Therefore, an enhancement of superconductivity owing to a mechanism similar to that in the ladders cannot be expected. Also, in the momentum-space picture, the incipient band situation, which is characteristic of multiband systems like the ladders, does not take place in the layered cuprates because they are essentially single-band systems.

From the above consideration, one may intuitively expect that uniaxially compressing the two-leg ladder cuprate in the rung direction would enhance superconductivity because a shorter $\mathrm{Cu}-\mathrm{Cu}$ distance in the rung direction is likely to increase the electron hopping in that direction. In the present study, we explore how the electron hoppings of the two-leg ladder cuprate are affected when uniaxial compression or tension is applied to the lattice in the leg or rung directions and investigate its consequences to superconductivity. For simplicity, we consider the two-leg ladder cuprate without the chains; that is, $\mathrm{SrCu}_{2} \mathrm{O}_{3}$ [31], although this material is known to be difficult to dope with carriers. We surprisingly find that the ratio $t_{r} / t_{l}$, where $t_{r}\left(t_{l}\right)$ is the nearest-neighbor hopping in the rung (leg) direction, is enhanced when the lattice is compressed in the leg direction or stretched in the rung direction. This counterintuitive manner of the hopping variation can be attributed to the on-site hybridization between $\mathrm{Cu} d_{x^{2}-y^{2}}$ and $\mathrm{Cu} 4 s$ orbitals, which arises due to the low symmetry of the lattice [32]. Due to such variation of the hoppings, we find that the superconducting transition temperature $T_{c}$ is enhanced when the lattice is compressed in the leg direction, which is opposed to an intuitive expectation. The effect is expected to be strong, especially in the electron-doped regime.

The model construction of $\mathrm{SrCu}_{2} \mathrm{O}_{3}$ is performed as follows: We take the lattice constant determined experimentally [33] as a reference and assume crystal structures compressed or stretched by a certain amount in the leg or rung directions. We determine the internal coordinates for these crystal structures through structural optimization and calculate the electronic band structure, using the Perdew-BurkeErnzerhof parametrization of the generalized gradient approximation (PBE-GGA) [34] and the projector augmented wave method [35] as implemented in the VASP code [36-39]. Plane-wave cutoff energy and the $k$-meshes were taken as 


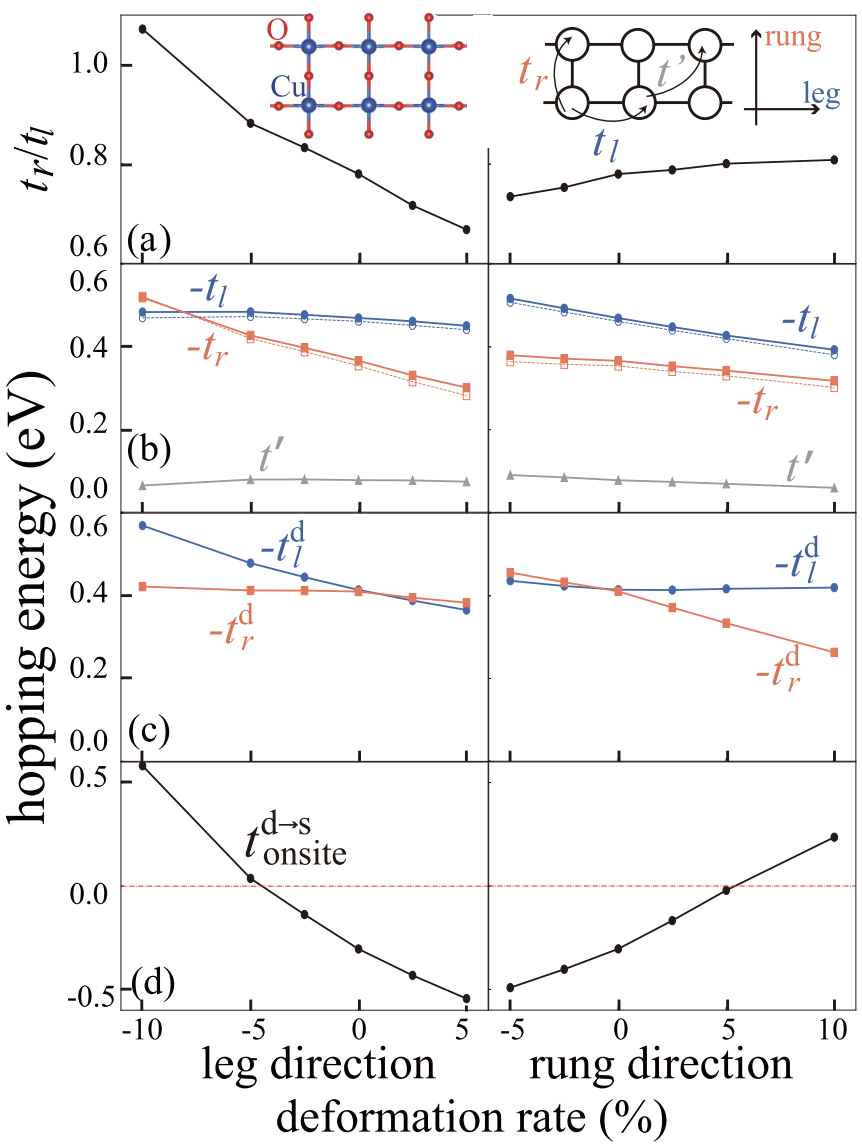

FIG. 1. The variation of the hoppings against uniaxial deformation in the leg (left panels) or rung (right panels) directions. We take the tension (+) or compression (-) rate as the horizontal axis. (a) The ratio $t_{r} / t_{l}$. (b) $t_{l}, t_{r}$, and $t^{\prime}$ of the composite- $d$ model (solid lines) and the $d$-s model estimation (see text) of $t_{l}$ and $t_{r}$ (dashed lines). (c) $t_{l}^{d}$ and $t_{r}^{d}$ in the $d$-s model. (d) $t_{\text {onsite }}^{d \rightarrow s}$ in the $d$-s model.

$550 \mathrm{eV}$ and $10 \times 10 \times 10$, respectively. We then extract the Wannier functions [40,41] from the calculated band structures by using the WANNIER90 [42] code, which gives the tightbinding hoppings and on-site energies $t_{i}^{\alpha \beta}$, where $i$ and $\alpha, \beta$ denote the lattice vectors and the orbitals, respectively. The tight-binding model in momentum space is obtained in the form $\varepsilon_{\alpha \beta}(\boldsymbol{k})=\sum_{i}^{N} t_{i}^{\alpha \beta} \exp \left(i \boldsymbol{k} \cdot \Delta \boldsymbol{r}_{i}\right)$, where we take $N=621$ lattice vectors $\Delta \boldsymbol{r}_{i}$.

Here, we first construct a two-orbital model where we explicitly consider the $d_{x^{2}-y^{2}}$ orbitals centered at the two Cu sites in a unit cell. These $d_{x^{2}-y^{2}}$ Wannier orbitals actually consist of a mixture of $\mathrm{Cu} d_{x^{2}-y^{2}}$, oxygen $2 p$ and, also, as explained later, $\mathrm{Cu} 4 s$ atomic orbitals. Throughout the paper, the oxygen orbitals are considered only implicitly, but later the $4 s$ orbital shall be explicitly taken into account. In the sense that the $d_{x^{2}-y^{2}}$ Wannier orbital here implicitly contains the $4 s$ orbital, this two-orbital model will be referred to as the "composite- $d$ model." From this model, we estimate the nearest-neighbor hoppings in the leg $\left(t_{l}\right)$ and rung $\left(t_{r}\right)$ directions, and also the next-nearest-neighbor diagonal hopping $t^{\prime}$ [see inset of Fig. 1(a)]. In Fig. 1(b) (solid lines), we present the variation of the hoppings $t_{l}, t_{r}$, and $t^{\prime}$ upon compressing or stretching the lattice in the leg or rung directions, and in Fig. 1(a) the variation of the ratio $t_{r} / t_{l}$. In contrast to an intuitive expectation, $t_{r} / t_{l}$ increases when the lattice is compressed in the leg direction or stretched in the rung direction.

To understand the origin of this variation of the hoppings against the lattice deformation, we now construct a model which explicitly takes into account not only the $3 d_{x^{2}-y^{2}}$ Wannier orbital but also the $\mathrm{Cu} 4 s$ orbital, which was implicitly taken into account in the composite- $d$ model. Note that the $3 d_{x^{2}-y^{2}}$ Wannier orbital in this model still implicitly takes into account the oxygen $2 p$ orbital as in the composite- $d$ model. This model will be called the $d$-s model. In fact, it has been known that the $4 s$ orbital hybridizes with $d_{x^{2}-y^{2}}$ to give rise to an appreciable diagonal hopping in the cuprates [43-46]. For the ladder structure in particular, it was pointed out in Ref. [32] that the anisotropy of the $4 s$-orbital-related hoppings is the origin of the anisotropy of the effective $d$ - $d$ hoppings in the leg and rung directions. In Fig. 1(c), we plot the hopping between the nearest neighbor $d_{x^{2}-y^{2}}$ orbitals in the leg $\left(t_{l}^{d}\right)$ and rung $\left(t_{r}^{d}\right)$ directions [47]. Now these hoppings behave as intuitively expected; namely, $t_{l}^{d}$ becomes large when the lattice is compressed in the leg direction, and $t_{r}^{d}$ is reduced when the lattice is stretched in the rung direction. Similarly, we find that the nearest-neighbor hoppings between $4 s$ and $d_{x^{2}-y^{2}}\left(t_{l, r}^{s \rightarrow d}\right)$ behave as intuitively expected under lattice deformation (not shown). We therefore expect that the hoppings between the $d_{x^{2}-y^{2}}$ orbitals via the $4 s$ orbital (see Fig. 2) play a crucial role in the counterintuitive lattice deformation dependence of the hoppings in the composite- $d$ model. We estimate the $d \rightarrow s \rightarrow d$ hopping using second-order perturbation theory as

$$
t_{l, r}^{d \rightarrow s \rightarrow d}=\frac{t_{\text {onsite }}^{d \rightarrow s} t_{l, r}^{s \rightarrow d}}{\varepsilon_{d}-\varepsilon_{s}},
$$

where $t_{\text {onsite }}^{d \rightarrow s}$ is the $d_{x^{2}-y^{2}}$ to $4 s$ hopping within the same $\mathrm{Cu}$ site, $t_{l, r}^{s \rightarrow d}$ is the nearest neighbor $4 s$ to $d_{x^{2}-y^{2}}$ hopping in the leg or rung directions, and $\varepsilon_{d, s}$ is the on-site energy of the $d_{x^{2}-y^{2}}$ or $4 s$ orbitals. Perturbation theory is expected to work since $\left|\varepsilon_{d}-\varepsilon_{s}\right| \approx 6 \mathrm{eV}$ is fairly large. Contribution from all possible equivalent paths are added up, and added to $t_{l, r}^{d}$, which gives the dashed-line plots in Fig. 2. As seen in this plot, the $d-s$ model estimation almost perfectly reproduces the composite- $d$ results, which confirms the view that the origin of the counterintuitive variation of $t_{r}$ and $t_{l}$ is the hopping path $d \rightarrow s \rightarrow d$ (we have also checked that contributions coming from other paths that involve the $4 s$ orbital are very small).

To further understand intuitively the contribution from the $d \rightarrow s \rightarrow d$ path, we focus on $t_{\text {onsite }}^{d \rightarrow s}$, plotted in Fig. 1(d), which changes sign upon lattice deformation. This sign change can be intuitively understood from the upper panels of Fig. 2. Namely, when the nearest-neighbor $\mathrm{Cu}-\mathrm{Cu}$ distance in the leg direction $a_{l}$ is long, the widely spread $4 s$ orbital is elongated in the leg direction, while the more localized $d_{x^{2}-y^{2}}$ orbital is less deformed. In this case, the on-site hopping is dominated by the longitudinal portion of the $d_{x^{2}-y^{2}}$ wave function, so that $t_{\text {onsite }}^{d \rightarrow s}<0$, taking the phase of the orbitals as depicted in the figure (note that the sign of a hopping is the opposite to that of the multiplication of the signs of the wave function of the initial and final orbitals because of the negative potential energy felt by the electrons). Similarly, when the 
(a)

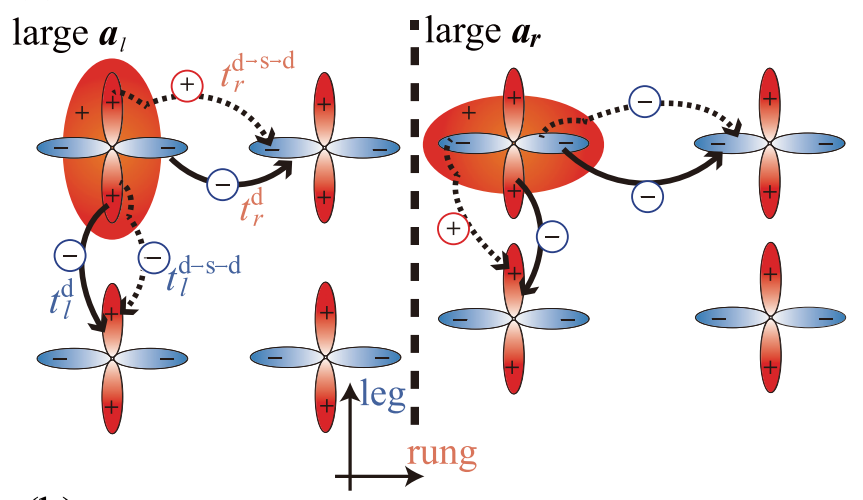

(b)

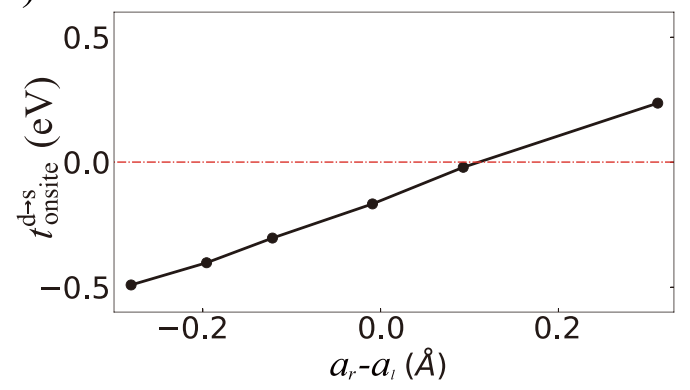

FIG. 2. (a) Schematic image of the hoppings of the $d$-s model in the large $a_{l}$ (left) and large $a_{r}$ (right cases). + and - enclosed by circles indicate the sign of the hoppings. Red (blue) portion of the wave functions has positive (negative) sign. Adding $t_{l, r}^{d \rightarrow s \rightarrow d}$ to $t_{l, r}^{d}$ explains the hopping variation in the composite- $d$ model against the lattice deformation. (b) The on-site hopping from $\mathrm{Cu} 4 s$ to $\mathrm{Cu} d_{x^{2}-y^{2}}$ orbitals in the $d$-s model, plotted against the difference between the nearest-neighbor distances in the leg and rung directions.

nearest-neighbor $\mathrm{Cu}-\mathrm{Cu}$ distance in the rung direction $a_{r}$ is long and the $4 s$ orbital is elongated in the rung direction, $t_{\text {onsite }}^{d \rightarrow s}>0$. This tendency is confirmed in the calculation result shown in the lower panel of Fig. 2. When $a_{l}$ is large and hence $t_{\text {onsite }}^{d \rightarrow s}<0$, from Eq. (1), $t_{r}^{d \rightarrow s \rightarrow d}>0$ and $t_{l}^{d \rightarrow s \rightarrow d}<0$ because $t_{r}^{s \rightarrow d}>0, t_{l}^{s \rightarrow d}<0$, and $\varepsilon_{d}-\varepsilon_{s}<0$. Similarly, when $a_{r}$ is large and hence $t_{\text {onsite }}^{d \rightarrow s}>0$, the sign of the indirect hoppings becomes the opposite as $t_{r}^{d \rightarrow s \rightarrow d}<0$ and $t_{l}^{d \rightarrow s \rightarrow d}>0$ [48]. Adding $t_{l, r}^{d \rightarrow s \rightarrow d}$ to the negative $t_{l, r}^{d}$ explains the counterintuitive variation of the hoppings in the composite- $d$ model against the lattice deformation.

We now move on to the analysis of superconductivity. To take into account the correlation effects that induce superconductivity, we add the on-site interaction $U$ term to the composite- $d$ model, where we take an on-site repulsion of $U=3 \mathrm{eV}$, which is a typical value for the cuprates [49]. The many-body study of this model is performed within the fluctuation exchange approximation (FLEX) [50]. We obtain the renormalized Green's function by solving Dyson's equation in a self-consistent calculation. The obtained Green's function and the pairing interaction mediated mainly by spin fluctuations are plugged into the linearized Eliashberg equation. The superconducting transition temperature $T_{c}$ is determined as the temperature where the eigenvalue of the Eliashberg equation reaches unity. In the FLEX calculation, $32 \times 32 \times 4$ $\left(k_{x}, k_{y}, k_{z}\right)$-meshes were taken.

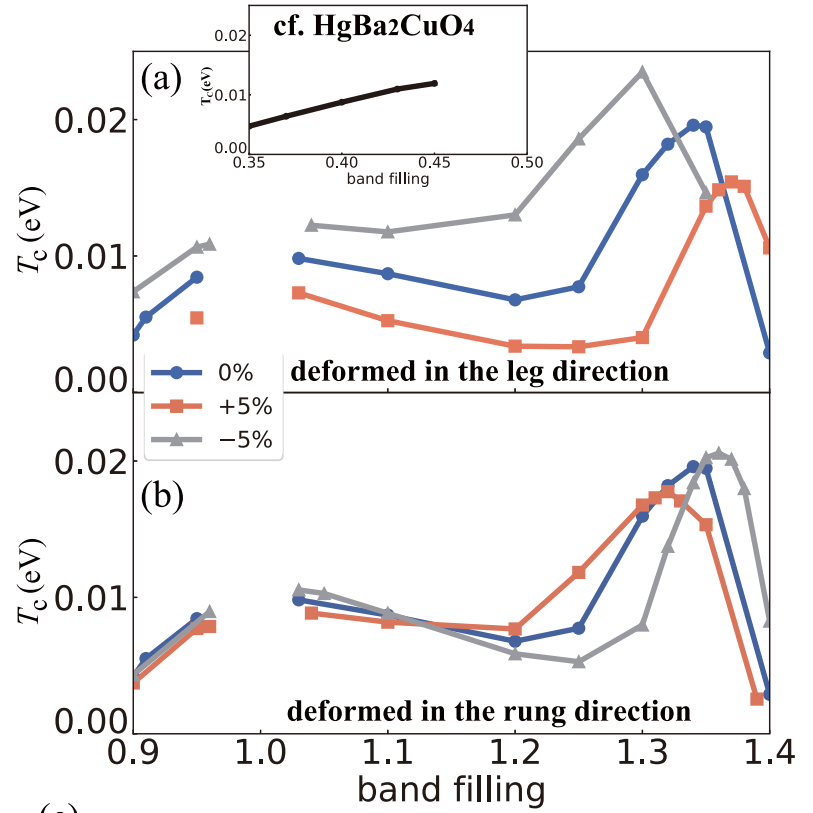

(c)

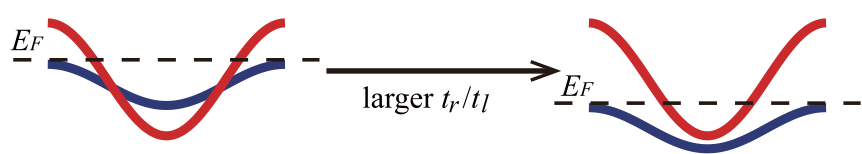

FIG. 3. The FLEX result of the superconducting transition temperature calculated for the composite- $d$ model against lattice deformation in the (a) leg or (b) rung directions. Inset of (a) shows a similar calculation result for the single orbital model of $\mathrm{HgBa}_{2} \mathrm{CuO}_{4}$. (c) A schematic image of the relative shift of the bonding and antibonding bands as $t_{r} / t_{l}$ increases.

Before going into the results, we comment on the $T_{c}$ obtained in the present calculation. The FLEX calculation for the Hubbard model on ladder-type lattice is often performed for a two-dimensional lattice where the ladders are weakly coupled [51] because a single-ladder system is a purely onedimensional system, where one might question the validity of perturbational approaches such as FLEX. Here, however, we perform the FLEX calculation for a purely-one-dimensional lattice since $T_{c}$ calculated in the present formalism barely depends on whether the weak ladder-ladder coupling is considered. To be more strict, a finite $T_{c}$ should not exist even for two-dimensional systems; finite $T_{c}$ is obtained for twodimensional as well as one-dimensional systems because a mean-field approximation is adopted. However, it was shown that introducing a weak three-dimensional coupling between two-dimensional layers also barely affects the $T_{c}$ in the FLEX calculation [52]. This further justifies our approach of applying FLEX to a single-ladder system. Another point that should be noted is that we do not calculate $T_{c}$ near half filling, where FLEX cannot sufficiently incorporate the strong correlation effects accompanying the Mott transition. In fact, taking into account the strong correlation effects should reduce $T_{c}$ near half filling, as shown in previous studies for the ladder [53] and two-dimensional systems [54].

In the upper panels of Fig. 3, we plot the superconducting transition temperature against the band filling $n$ (= number of electrons/number of sites) for the cases when the lattice is 
compressed or stretched by $5 \%$ in the leg or rung directions. A common feature in all cases is the double local maximum of $T_{c}$, one around half filling, and another at $n>1$, i.e., in the electron-doped regime. $T_{c}$ is enhanced near half filling due to the enhancement of electron correlation. A prominent feature peculiar to the ladder-type lattice is the rather high $T_{c}$ in the electron-doped regime. The $T_{c}$ maximum in this regime is about twice as high as that of the $T_{c}$ calculated in the same way for a $100 \mathrm{~K}$ cuprate superconductor $\mathrm{HgBa}_{2} \mathrm{CuO}_{4}$, shown in the inset of Fig. 3. To understand this $T_{c}$ maximum, we introduce the tight-binding band dispersion of the two-leg ladder given as $E_{ \pm}(k)=t_{l}\left[2\left(1 \mp \frac{t^{\prime}}{t_{l}}\right) \cos (k) \pm \frac{t_{r}}{t_{l}}\right]$, where and + in $E_{ \pm}(k)$ stand for bonding and antibonding bands, respectively, and $-1<t^{\prime} / t_{l}<0$ (appropriate for the cuprates) makes the bonding band narrower than the antibonding band. In the previous studies $[11,13]$, it was shown that $T_{c}$ is strongly enhanced when the Fermi level is raised by electron doping (the necessity of about $30 \%$ electron doping was suggested in Ref. [11]) so that it lies just above the top of the bonding band. There, this was considered as an example of superconductivity enhanced in systems with coexisting wide and narrow bands, when the Fermi level is positioned in the vicinity of the narrow-band edge; namely, when the narrow band is "incipient" [17-26].

Now, when the lattice is compressed in the leg direction or stretched in the rung direction, the ratio $t_{r} / t_{l}$ increases (while $t^{\prime} / t_{l}$ is barely affected) as we have seen, so that the bonding band is lowered relatively to the antibonding band, as depicted schematically in the lower panel of Fig. 3. Hence, less electron is required for the Fermi level to reach the vicinity of the bonding band top. This is the reason why the $T_{c}$ maximum moves toward the less-electron-doped regime in these cases. Especially when the lattice is compressed in the leg direction, the maximum $T_{c}$ itself is enhanced because the electron correlation effect becomes stronger as the band filling approaches half filling. On the other hand, when the lattice is stretched in the rung direction, although $T_{c}$ is maximized in the lesselectron-doped regime, the maximum $T_{c}$ is suppressed. This is because $\left|t_{l}\right|$ decreases, leading to the reduction of the energy scale, opposed to when compressed in the leg direction, where $t_{l}$ barely varies.

We now extract the $4 s$ orbital effect by considering a hypothetical two-orbital model where the $4 s$ orbitals are removed "by hand" from the $d-s$ model. $T_{c}$ calculated for this " $d$ only" model, adopting the same $U=3 \mathrm{eV}$, against the band filling for the same lattice deformation as in the composite- $d$ model is shown in Fig. 4. The trend is almost completely the opposite compared with the composite- $d$ model; namely, the local $T_{c}$ maximum is reduced and moves toward the more-electron-doped regime when the lattice is compressed in the leg direction or stretched in the rung direction. This is because the counterintuitive variation of $t_{r} / t_{l}$ is lost in the absence of $4 s$. Therefore, compressing the lattice in the leg direction or stretching it in the rung direction simply suppresses $t_{r} / t_{l}$, requiring large amount of doped electrons for the bonding band to be incipient. These results conversely reveal the crucial role played by the implicitly considered $4 s$ orbital in the composite- $d$ model [55].

The quantitative analysis on superconductivity in our study is based on FLEX, which is a kind of weak-coupling

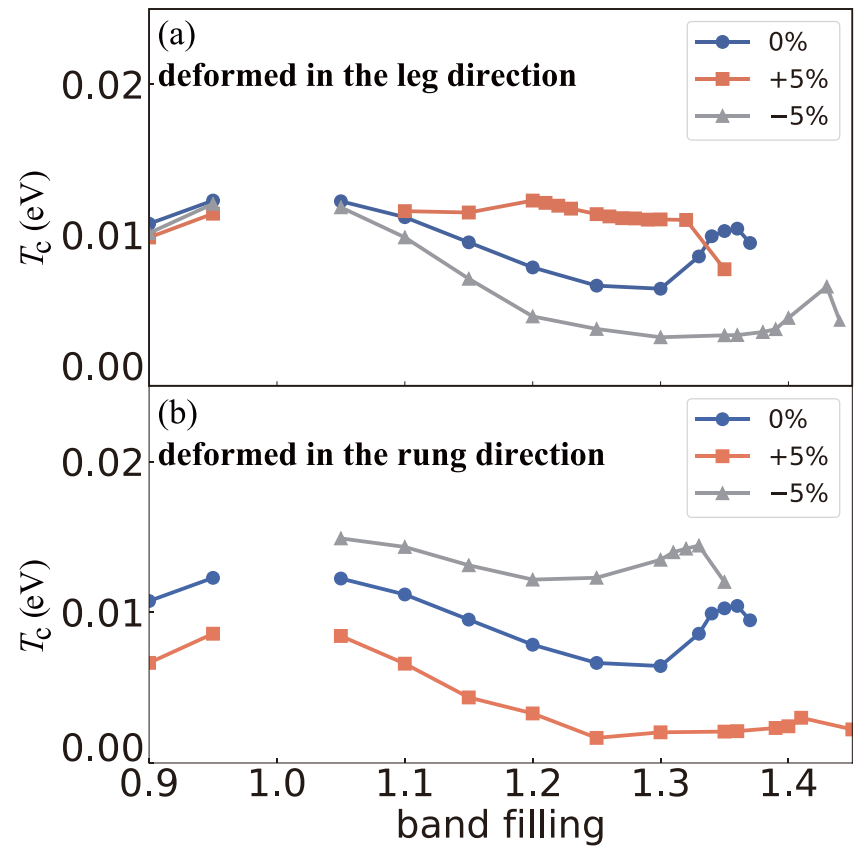

FIG. 4. Plots similar to Fig. 3 for the hypothetical " $d$-only" two-orbital model, obtained by removing the $4 s$ orbitals from the $d$-s model. Deformed in the (a) leg or (b) rung directions.

approach. However, the main conclusion drawn here, that longitudinal compression is favorable for superconductivity, is qualitatively unaffected in a wider scope. Namely, the tendency of enhanced superconductivity with larger $t_{r}$ (for a fixed $t_{l}$ ) has been pointed out in a previous density-matrix renormalization-group study [6], which is suitable for treating strong correlation effects. Moreover, in the large- $U$ limit, the spin-spin couplings in the leg and rung directions are given as $J_{l}=4 t_{l}^{2} / U$ and $J_{r}=4 t_{r}^{2} / U$, respectively. The compression of the lattice in the leg direction enhances $t_{r}$ and hence $J_{r}$ (while keeping $t_{l}$ and $J_{l}$ almost unchanged), from which we expect superconductivity to be enhanced based on the previous studies on the $t-J$ model on a ladder-type lattice $[1,7-$ 10]. Although we cannot be certain about how the "incipient band" situation affects superconductivity in the $t-J$ model, we do expect a larger effect in the electron-doped regime than in the hole-doped regime due to the larger density of states in the former.

The relevance of the present study to experiments is of great interest. In fact, it was found in Ref. [56] that applying uniaxial pressure to $(\mathrm{Sr}, \mathrm{Ca}){ }_{14} \mathrm{Cu}_{24} \mathrm{O}_{41}$ in the leg direction results in an enhancement of $T_{c}$ compared with the case when hydrostatic pressure is applied [5,57], although the quantitative correspondence between theory and experiment is not clear.

To summarize, we have investigated how superconductivity in the ladder-type cuprates is affected through modification of the electronic structure when uniaxial compression or tension is applied. It is found that the ratio $t_{r} / t_{l}$ is enhanced when the lattice is compressed in the leg direction or stretched in the rung direction. This counterintuitive manner of the hopping variation is attributed to the on-site hybridization between $\mathrm{Cu} d_{x^{2}-y^{2}}$ and $\mathrm{Cu} 4 s$ orbitals, which varies as the $4 s$ orbital is deformed through the lattice deformation. Due to 
such variation of the hoppings, $T_{c}$ is enhanced when the lattice is compressed in the leg direction, as opposed to an intuitive expectation. The effect is expected to be strong, especially in the electron-doped regime, where the Fermi level approaches the top of the bonding band.
We acknowledge Daisuke Ogura, Karin Matsumoto, Masayuki Ochi, and Hidetomo Usui for valuable discussions. This study was supported by JSPS KAKENHI Grant No. JP18H01860.
[1] E. Dagotto, J. Riera, and D. Scalapino, Phys. Rev. B 45, 5744(R) (1992).

[2] T. M. Rice, S. Gopalan, and M. Sigrist, Europhys. Lett. 23, 445 (1993).

[3] E. Dagotto and T. M. Rice, Science 271, 618 (1996).

[4] For a review, see, T. Vuletić, B. Korin-Hamzić, T. Ivek, S. Tomić, B. Gorshunov, M. Dressel, and J. Akimitsu, Phys. Rep. 428, 169 (2006).

[5] M. Uehara, T. Nagata, J. Akimitsu, H. Takahashi, N. Môri, and K. Kinoshita, J. Phys. Soc. Jpn. 65, 2764 (1996).

[6] R. M. Noack, N. Bulut, D. J. Scalapino, and M. G. Zacher, Phys. Rev. B 56, 7162 (1997).

[7] M. Troyer, H. Tsunetsugu, T. M. Rice, J. Riera, and E. Dagotto, Phys. Rev. B 48, 4002 (1993).

[8] H. Tsunetsugu, M. Troyer, and T. M. Rice, Phys. Rev. B 51, 16456 (1995).

[9] K. Sano, J. Phys. Soc. Jpn. 65, 1146 (1996).

[10] C. A. Hayward and D. Poilblanc, Phys. Rev. B 53, 11721 (1996).

[11] K. Kuroki, T. Higashida, and R. Arita, Phys. Rev. B 72, 212509 (2005).

[12] D. Ogura, H. Aoki, and K. Kuroki, Phys. Rev. B 96, 184513 (2017).

[13] K. Matsumoto, D. Ogura, and K. Kuroki, Phys. Rev. B 97, 014516 (2018).

[14] D. Ogura, Theoretical Study of Electron Correlation Driven Superconductivity in Systems with Coexisting Wide and Narrow Bands (Springer, Singapore, 2019).

[15] K. Matsumoto, D. Ogura, and K. Kuroki, J. Phys. Soc. Jpn. 89, 044709 (2020).

[16] D. Kato and K. Kuroki, Phys. Rev. Research 2, 023156 (2020).

[17] F. Wang, F. Yang, M. Gao, Z.-Y. Lu, T. Xiang, and D.-H. Lee, Europhys. Lett. 93, 57003 (2011).

[18] X. Chen, S. Maiti, A. Linscheid, and P. J. Hirschfeld, Phys. Rev. B 92, 224514 (2015).

[19] P. J. Hirschfeld, M. M. Korshunov, and I. I. Mazin, Rep. Prog. Phys. 74, 124508 (2011).

[20] Y. Bang, New J. Phys. 16, 023029 (2014).

[21] Y. Bang, New J. Phys. 18, 113054 (2016).

[22] Y. Bang, Sci. Rep. 9, 3907 (2019).

[23] A. Charnukha, D. V. Evtushinsky, C. E. Matt, N. Xu, M. Shi, B. Büchner, N. D. Zhigadlo, B. Batlogg, and V. Borisenko, Sci. Rep. 5, 18273 (2015).

[24] H. Miao, T. Qian, X. Shi, P. Richard, T. K. Kim, M. Hoesch, L. Y. Xing, X.-C. Wang, C.-Q. Jin, J.-P. Hu, and H. Ding, Nat. Commun. 6, 6056 (2015).

[25] A. A. Kordyuk, Low Temp. Phys. 44, 477 (2018).

[26] T. A. Maier, V. Mishra, G. Balduzzi, and D. J. Scalapino, Phys. Rev. B 99, 140504(R) (2019).
[27] K. Kobayashi, M. Okumura, S. Yamada, M. Machida, and H. Aoki, Phys. Rev. B 94, 214501 (2016).

[28] T. Misumi and H. Aoki, Phys. Rev. B 96, 155137 (2017).

[29] S. Sayyad, E. W. Huang, M. Kitatani, M.-S. Vaezi, Z. Nussinov, A. Vaezi, and H. Aoki, Phys. Rev. B 101, 014501 (2020).

[30] H. Aoki, arXiv:1912.04469.

[31] Z. Hiroi, M. Azuma, M. Takano, and Y. Bando, J. Solid State Chem. 95, 230 (1991).

[32] T. F. A. Müller, V. Anisimov, T. M. Rice, I. Dasgupta, and T. Saha-Dasgupta, Phys. Rev. B 57, R12655(R) (1998).

[33] K. Sparta, A. Löffert, C. Gross, W. Aßmus, and G. Roth, Z. Kristallogr. 221, 782 (2006).

[34] J. P. Perdew, K. Burke, and M. Ernzerhof, Phys. Rev. Lett. 77, 3865 (1996).

[35] G. Kresse and D. Joubert, Phys. Rev. B 59, 1758 (1999).

[36] G. Kresse and J. Hafner, Phys. Rev. B 47, 558(R) (1993).

[37] G. Kresse and J. Hafner, Phys. Rev. B 49, 14251 (1994).

[38] G. Kresse and J. Furthmüller, Comput. Mater. Sci. 6, 15 (1996).

[39] G. Kresse and J. Furthmüller, Phys. Rev. B 54, 11169 (1996).

[40] N. Marzari and D. Vanderbilt, Phys. Rev. B 56, 12847 (1997).

[41] I. Souza, N. Marzari, and D. Vanderbilt, Phys. Rev. B 65, 035109 (2001).

[42] A. A. Mostofi, J. R. Yates, Y.-S. Lee, I. Souza, D. Vanderbilt, and N. Marzari, Comput. Phys. Commun. 178, 685 (2008).

[43] O. K. Andersen, A. I. Liechtenstein. O. Jepsen, and F. Paulsen, J. Phys. Chem. Solids 56, 1573 (1995).

[44] E. Pavarini, I. Dasgupta, T. Saha-Dasgupta, O. Jepsen, and O. K. Andersen, Phys. Rev. Lett. 87, 047003 (2001).

[45] H. Sakakibara, H. Usui, K. Kuroki, R. Arita, and H. Aoki, Phys. Rev. B 85, 064501 (2012).

[46] H. Sakakibara, H. Usui, K. Kuroki, R. Arita, and H. Aoki, Phys. Rev. Lett. 105, 057003 (2010).

[47] Since the $d_{x^{2}-y^{2}}$ Wannier orbital implicitly considers the oxygen $2 p$ orbitals, the hoppings $t_{l}^{d}$ and $t_{r}^{d}$ here mainly originate from the path $\mathrm{Cu} d_{x^{2}-y^{2}} \rightarrow \mathrm{O} 2 p \rightarrow \mathrm{Cu} d_{x^{2}-y^{2}}$ in terms of the atomic orbitals.

[48] In the large- $a_{r}$ limit, all of the hoppings in the rung direction would evidently converge to zero, while the hoppings in the leg direction remains finite. Hence, for a fixed $a_{l}$, there should be an $a_{r}$ (in the unrealistically-large- $a_{r}$ regime) where $t_{r} / t_{l}$ would be maximized.

[49] S. W. Jang, H. Sakakibara, H. Kino, T. Kotani, K. Kuroki, and M. J. Han, Sci. Rep. 6, 33397 (2016).

[50] N. E. Bickers, D. J. Scalapino, and S. R. White, Phys. Rev. Lett. 62, 961 (1989).

[51] See, e.g., Ref. [13] and references therein.

[52] R. Arita, K. Kuroki, and H. Aoki, J. Phys. Soc. Jpn. 69, 1181 (2000). 
[53] I. Ichinose and T. Matsui, Phys. Rev. B 57, 13790 (1998).

[54] For a review, see, e.g., P. A. Lee, N. Nagaosa, and X.-G. Wen, Rev. Mod. Phys. 78, 17 (2006).

[55] See Supplemental Material at http://link.aps.org/supplemental/ 10.1103/PhysRevResearch.2.022055 for further analysis on the $d$-only model.
[56] D. Mohan Radheep, R. Thiyagarjan, S. Esakkimuthu, G. Deng, E. Pomjakushina, C. L. Prajapat, G. Ravikumar, K. Conder, G. Baskaran, and S. Arumugam, arXiv:1303.0921.

[57] T. Nagata, M. Uehara, J. Goto, J. Akimitsu, N. Motoyama, H. Eisaki, S. Uchida, H. Takahashi, T. Nakanishi, and N. Môri, Phys. Rev. Lett. 81, 1090 (1998). 